\title{
Texture Analysis in Ultrasound Images of Carotid Plaque Components of Asymptomatic and Symptomatic Subjects
}

\author{
Christos P. Loizou ${ }^{1, *}$, Marios Pantziaris ${ }^{2}$, Marilena Theofilou ${ }^{3}$, \\ Takis Kasparis ${ }^{3}$, and Efthivoulos Kyriakou ${ }^{4}$ \\ ${ }^{1}$ Department of Computer Science, Intercollege, P.O. Box 51604, CY-3507, \\ Limassol, Cyprus \\ panloicy@logosnet.cy.net \\ ${ }^{2}$ Cyprus Institute of Neurology and Genetics, Nicosia, Cyprus \\ pantzariecing.ac.cy \\ ${ }^{3}$ Cyprus University of Technology, Departement of Electical, \\ Computer Engineering and Informatics, Limassol, Cyprus \\ takis.kasparis@cut.ac.cy \\ ${ }^{4}$ Dept. of Computer Science and Engineering, Frederick University Cyprus, \\ Limassol, Cyprus \\ e.kyriacoulfrederick.ac.cy
}

\begin{abstract}
There are indications that the texture of certain components of atherosclerotic carotid plaques in the common carotid artery (CCA), obtained by high resolution ultrasound imaging, may have additional prognostic implication for the risk of stroke. The objective of this study was to perform texture analysis of the middle component of atherosclerotic carotid plaques in 230 CCA plaque ultrasound images (115 asymptomatic and 115 symptomatic). These were manually delineated by a neurovascular expert after normalization and despeckle filtering using the linear despeckle filter (DsFlsmv). Texture features were extracted from the middle plaque component. We found statistical significant differences for some of the texture features extracted, between asymptomatic and symptomatic subjects. The results showed that it may be possible to identify a group of patients at risk of stroke (asymptomatic versus symptomatic) based on texture features extracted from the middle component of the atherosclerotic carotid plaque in ultrasound images of the CCA.
\end{abstract}

Keywords: Atherosclerotic carotid plaque components, texture analysis, ultrasound image, carotid artery.

\section{Introduction}

Cardiovascular disease (CVD) is the third leading cause of death and major disability in the world and is the consequence of increased atherosclerosis in the walls of the common-carotid artery (CCA) [1]. Atherosclerosis is a buildup on the artery walls and

* Corresponding author.

H. Papadopoulos et al. (Eds.): AIAI 2013, IFIP AICT 412, pp. 282-291, 2013.

(C) IFIP International Federation for Information Processing 2013 
is the main reason leading to CVD and can result to heart attack, and stroke [1], [2]. It is a disease of the large and medium size arteries, and it is characterized by plaque formation (see also Fig. 1a) due to progressive intimal accumulation of lipid, protein, and cholesterol esters in the blood vessel wall [1]-[3], which reduces blood flow significantly. The risk of stroke increases with the severity of carotid stenosis and is reduced after carotid endarterectomy [2], [3]. There have been clinical indications that different components of the atherosclerotic carotid plaque of the CCA may contribute differently to the development of the risk of stroke. The objective of this study was to investigate the middle component of the atherosclerotic carotid plaque between asymptomatic and symptomatic subjects at risk of atherosclerosis, based on texture features analysis extracted from this area, and provide information that might indicate the importance of this structure in the development of stroke or of future neurological events.

There are only few studies reported in the literature, for the segmentation of the CCA plaque from ultrasound images [4], where the manual delineation of the plaques was used to be the golden standard [2], [3]. Recently a full automated system for the full segmentation of the CCA in ultrasound images based on snakes was proposed [5], as well as a system for the video segmentation of the CCA plaque [6]. There is only one study found in the literature [7], where the association between the presence of juxtaluminal hypoechoic areas and clinical symptoms was investigated. Furthermore, a relatively small number of plaque and classification studies based on B-mode ultrasonic imaging have been proposed and used in several cross-sectional and longitudinal natural history studies [8]-[10], attempting to correlate ultrasonic plaque features with the development of neurological events. More specifically, in [8] an automated segmentation method was proposed by our group based on image normalization, despeckle filtering and initial plaque contour estimation for segmenting the atherosclerotic carotid plaque from ultrasound images of the CCA. In [9], texture features were extracted from asymptomatic and symptomatic subjects at risk of atherosclerosis and features were found which may classify these groups of subjects. Finally, in [10] morphological features extracted from plaques areas were used for classifying plaques in asymptomatic and symptomatic.

In this study, we use the manual segmentations from a neurovascular expert, for investigating the middle component of the atherosclerotic carotid plaque in ultrasound images of the CCA (see also Fig. 1) in 115 asymptomatic and 115 symptomatic subjects and, perform texture analysis of this structure. Our objective in this study was to estimate significant differences between the aforementioned groups of patients, which may help in the correct evaluation of the risk of stroke. The study showed that there are texture features that may be used to classify the two different groups of subjects as well as to follow up the progression of the atherosclerosis disease. The findings of this study may additionally be helpful in understanding the development of stroke in asymptomatic and/or asymptomatic subjects at risk of atherosclerosis. 


\section{Materials and Methods}

\subsection{Recording of Ultrasound CCA Plaque Images}

A total of 230 (115 asymptomatic and 115 symptomatic) B-mode longitudinal ultrasound images of the CCA bifurcation were acquired by the ATL HDI-3000 ultrasound scanner (Advanced Technology Laboratories, Seattle, USA) [11], equipped with a high linear array transducer (4-7 MHz), and were recorded digitally on a magneto optical drive, with a resolution of 768x576 pixels with 256 gray levels. Asymptomatic images were recorded from patients at risk of atherosclerosis in the absence of clinical symptoms. Symptomatic images were recorded from patients at risk of atherosclerosis, who already have developed clinical symptoms, such as a stroke or a transient ischemic attack (TIA). Digital images were resolution normalized

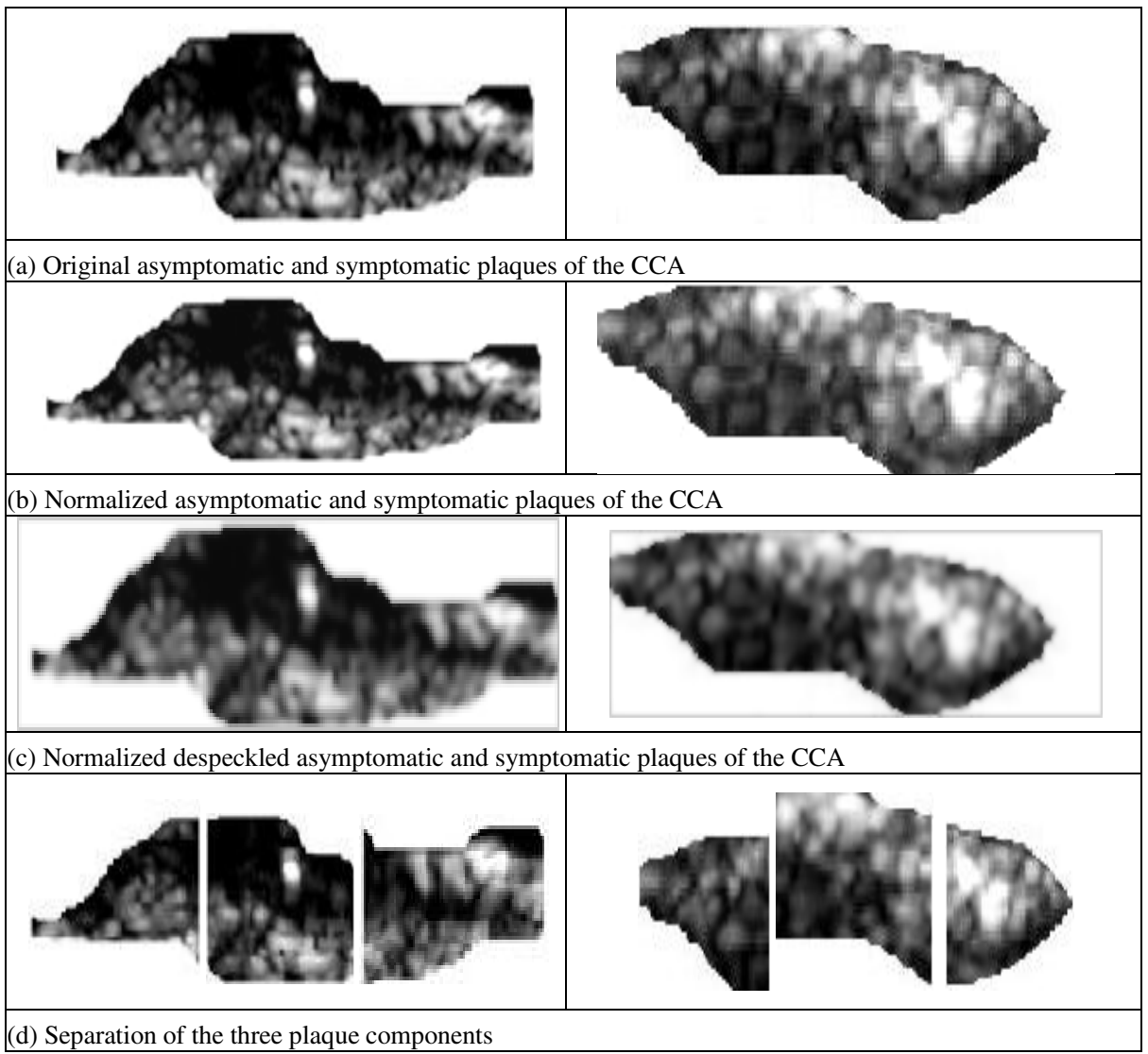

Fig. 1. a) Manually segmenetd ultrasound CCA plaque images of an asymptomatic (left colum) and a symptomatic subject (right column), b) Intensity normalised plaque images from a), c) Normalised and despeckled carotid plaque images with the filter DsFlsmv, d) Automatted segmentions of the three different plaque components. 
at 16.66 pixels $/ \mathrm{mm}$ (see ultrasound image normalization section). This was carried out due to the small variations in the number of pixels per $\mathrm{mm}$ of image depth (i.e. for deeply situated carotid arteries, image depth was increased and therefore digital image spatial resolution would have decreased) and in order to maintain uniformity in the digital image spatial resolution [8], [10]. The images were recorded at the Institute of Neurology and Genetics, Nicosia, Cyprus.

\subsection{Ultrasound Image Normalization}

Brightness adjustments of ultrasound images were carried out in this study based on the method introduced in [12], which improves image compatibility by reducing the variability introduced by different gain settings, different operators, different equipment, and facilitates ultrasound tissue comparability. Algebraic (linear) scaling of the images were manually performed by linearly adjusting the image so that the median gray level value of the blood was $0-5$, and the median gray level of the adventitia (artery wall) was 180-190 [12]. The scale of the gray level of the images ranged from 0-255. Thus the brightness of all pixels in the image was readjusted according to the linear scale defined by selecting the two reference regions. Further details of the proposed normalization method can be found in [4]-[10].

\subsection{Manual Segmentation and Separation of Plaques}

A neurovascular expert (coauthor Marios Pantziaris) manually delineated (using the mouse) the $230 \mathrm{CCA}$ plaque ultrasound images $(115$ symptomatic +115 asymptomatic). The plaques were used for normalization, speckle reduction filtering, and texture features extraction. The manual delineations were performed using a system implemented in Matlab (Math Works, Natick, MA) from our group. The delineations were performed between 1 and $2 \mathrm{~cm}$ proximal to the bifurcation of the CCA at the far wall [12] over a distance of $1.5 \mathrm{~cm}$ starting at a point $0.5 \mathrm{~cm}$ and ending at a point $2.0 \mathrm{~cm}$ proximal to the carotid bifurcation. The bifurcation of the CCA was used as a guide, and all measurements were made from that region. The plaque contours were saved in order to be used for the texture analysis (see subsection 2.5). All sets of manual segmentation measurements were performed by the expert in a blinded manner, both with respect to identifying the subject and delineating the image. The 230 CCA plaques were then automatically separated in three different equidistant components. The horizontal major axis of the segmented plaque was estimated and perpendicular lines (starting from the left component of the plaque) were selected, at the $33 \%$, and $66 \%$ of the plaque horizontal major axis (see also Fig. 1d), and thus separating the CCA plaques into three different components of equal major axis length sizes. The middle component of the plaque was then used for texture features analysis.

\subsection{Speckle Reduction Filtering (DsFlsmv)}

In this study the linear scaling filter (despeckle filter linear scaling mean varianceDsFlsmv) [13], utilizing the mean and the variance of a pixel neighborhood was used 
in order to filter the CCA ultrasound plaque images from multiplicative noise prior the texture features extraction. The filter may be described by a weighted average calculation using sub region statistics to estimate statistical measurements over $5 \times 5$ pixel windows applied for two iterations on each image. The DsFlsmv filter was applied on 440 CCA ultrasound images in [14], compared with 10 other different despeckle filters, and showed the best performance.

Table 1. Texture Features (mean $\pm \mathrm{IQR})$ Extracted from the Asymptomatic $(\mathrm{N}=115)$ and the Symptomatic $(\mathrm{N}=115)$ CCA Plaques for the Original and the Despeckled Images (- / -). Interquartile range values are given in parentheses $( \pm \mathrm{IQR})$

\begin{tabular}{|c|c|c|c|}
\hline $\begin{array}{l}\text { Texture } \\
\text { Features }\end{array}$ & Asymptomatic $(\mathrm{N}=115)$ & Symptomatic $(\mathrm{N}=115)$ & Wilcoxon test ${ }^{1}$ \\
\hline \multicolumn{4}{|c|}{ Statistical Features } \\
\hline Median & $58.7(39.1) / 49.5(50.1)$ & $39.7(34.9) / 28(40.7)$ & $0.007 / 0.005$ \\
\hline \multicolumn{4}{|c|}{ Spatial Gray Level Dependence matrix (SGLDM) } \\
\hline Contrast & $23(13) / 29(3)$ & $51(61) / 78(40)$ & $0.02 / 0.02$ \\
\hline IDM & $0.33(0.22) / 0.28(0.18)$ & $0.48(0.31) / 0.41(0.29)$ & $0.01 / 0.001$ \\
\hline SA & $117(77.8) / 132(81)$ & $79.5(70.3) / 96.5(71.7)$ & $0.006 / 0.001$ \\
\hline \multicolumn{4}{|c|}{ Gray level Difference Statistics (GLDS) } \\
\hline Entropy & $5.7(2.1) / 7.2(1.7)$ & $4.4(2.54) / 5.9(2.68)$ & $0.002 / 0.006$ \\
\hline ASM & $0.005(0.001) / 0.003(0.002)$ & $0.01(0.01) / 0.005(0.003)$ & $0.005 / 0.007$ \\
\hline \multicolumn{4}{|c|}{ Statistical Feature Matrix (SFM) } \\
\hline Coarseness & $16.4(5.2) / 36.8(23.1)$ & $7.2(5.2) / 21.4(16.4)$ & $0.001 / 0.03$ \\
\hline Complexity & $\begin{array}{c}29180(16832) / \\
99876(47230)\end{array}$ & $\begin{array}{c}25463(23123) / \\
07838(67293)\end{array}$ & $\begin{array}{c}0.001 / \\
0.004\end{array}$ \\
\hline \multicolumn{4}{|c|}{$\begin{array}{c}\text { Laws Texture Energy Measures } \\
\end{array}$} \\
\hline Energy LL & $\begin{array}{l}177502(55567) / \\
185517(59639)\end{array}$ & $\begin{array}{c}148720(94936) / \\
157578(97482) \\
\end{array}$ & $\begin{array}{c}0.013 / \\
0.005\end{array}$ \\
\hline \multicolumn{4}{|c|}{$\begin{array}{l}\text { Fractal Dimension (FD) } \\
\end{array}$} \\
\hline Radial Sum & $3723(1752) / 5140(1723)$ & $2831(1656) / 4367(1579)$ & $0.002 / 0.0001$ \\
\hline ASM & $3120(1402) / 3930(1645)$ & $2435(1533) / 3288(1394)$ & $0.001 / 0.002$ \\
\hline \multicolumn{4}{|c|}{ Grey Level Run Length (GLRL) } \\
\hline SRE & $0.17(0.01) / 0.15(0.01)$ & $0.16(0.01) / 0.14(0.009)$ & $0.0001 / 0.002$ \\
\hline LRE & $38.2(2.03) / 38.6(1.9)$ & $38.3(1.82) / 38.8(1.67)$ & $0.003 / 0.006$ \\
\hline
\end{tabular}

IDM: Inverse difference moment, SA: Sum average, ASM: Angular second moment, SRE: Short run emphasis, LRE: Long-run emphasis. ${ }^{1}$ Test Carried out at $\mathrm{p}<0.05$ between the asymptomatic and the symptomatic despeckled CCA plaques.

\subsection{Texture Analysis}

Texture provides useful information for the characterization of plaque images in the CCA [9]. In order to estimate textural characteristics extracted from the different plaque components, a total of 71 different texture features were extracted, where only the most significant are shown in this work. The following texture feature sets were used: (i) First Order Statistics (FOS) [9], [15]: a) mean, b) variance, c) median, d) 


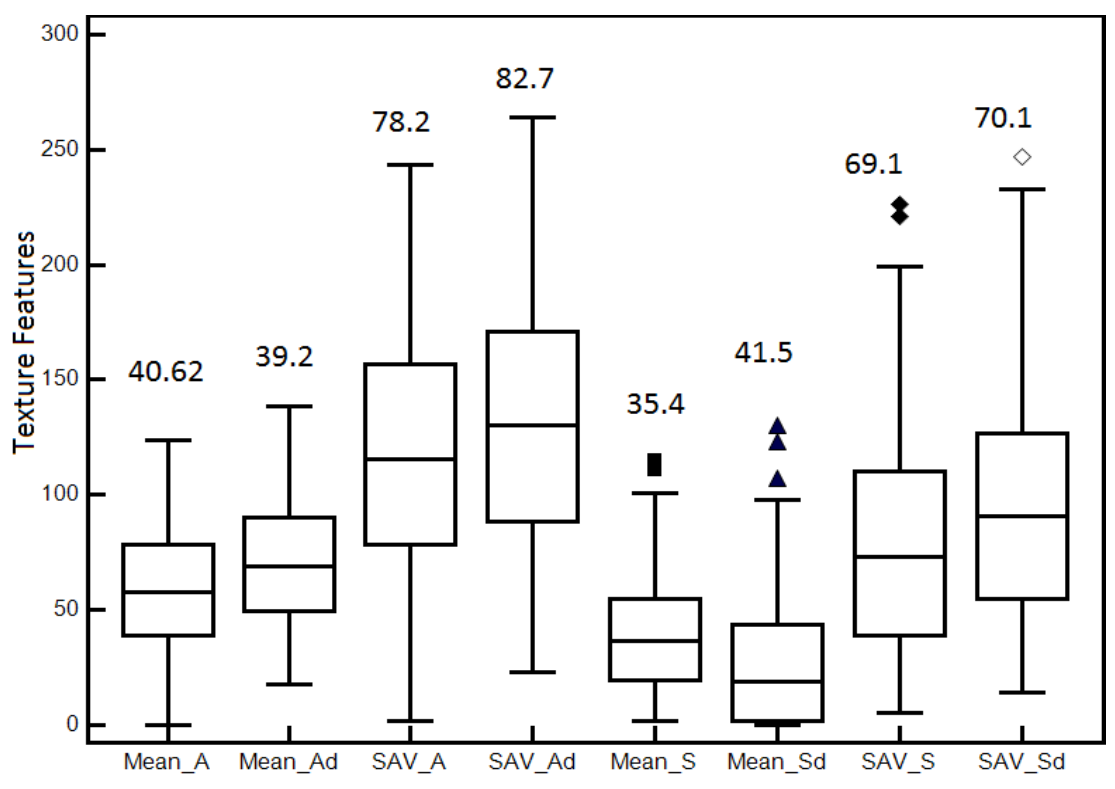

Fig. 2. Box plots for selected plaque texture features extracted from the middle component of the asymptomatic and symptomatic CCA plaques from all patients for the normalised despeckled images. We show the texture features: a) mean and sum average (SAV) for asymptomatic (_A) and symptomatic (_S) subjects as well as for original and despeclked (_d) images. In each plot we display the median, lower, and upper quartiles and confidence interval around the median. Straight lines connect the nearest observations within 1.5 of the IQR of the lower and upper quartiles. Unfilled triangles indicate possible outliers with values beyond the ends of the $1.5 \mathrm{x}$ IQR. IQR values are shown above each boxplot.

skewness, e) kurtosis, f) energy, and g) entropy. (ii) Spatial Gray Level Dependence Matrices (SGLDM) as proposed by Haralick et al. [15]: a) angular second moment, b) contrast, c) correlation, d) sum of squares variance (SOSV), e) inverse difference moment (IDM), f) sum average, g) sum variance, h) sum entropy, i) entropy, j) difference variance, $\mathrm{k}$ ) difference entropy, and 1) information measures of correlation. For a chosen distance $\mathrm{d}$ (in this work $\mathrm{d}=1$ was used) and for angles $\theta=0^{\circ}, 45^{\circ}, 90^{\circ}$, and $135^{\circ}$, we computed four values for each of the above texture measures. (iii) Gray Level Difference Statistics (GLDS) [16]: a) homogeneity, b) contrast, c) energy, d) entropy, and e) mean. The above features were calculated for displacements $\delta=(0,1)$, $(1,1),(1,0),(1,-1)$, where $\delta \equiv(\Delta x, \Delta y)$, and their mean values were taken. (iv) Neighborhood Gray Tone Difference Matrix (NGTDM) [17]: a) coarseness, b) contrast, c) busyness, d) complexity, and e) strength. (v) Statistical Feature Matrix (SFM) [18]: a) coarseness, b) contrast, c) periodicity, and d) roughness. (vi) Laws Texture Energy Measures (LTEM) [18]: LL-texture energy from LL kernel, EEtexture energy from EE-kernel, SS-texture energy from SS-kernel, LE-average texture energy from LE and EL kernels, ES-average texture energy from ES and SE kernels, and LS-average texture energy from LS and SL kernels. (vii) Fractal Dimension 
Texture Analysis (FDTA) [19]: The Hurst coefficients for dimensions 4, 3 and 2 were computed. (viii) Fourier Power Spectrum (FPS) [19]: a) radial sum, and b) angular sum. (ix) Gray-level run length (GLRL) [10]: a) Short run emphasis, b) long run emphasis, c) gray level distribution, d) run length distribution), e) run percentage), and f)-j) mean values of the GLRL group for a)-e).

\subsection{Statistical Analysis}

The Wilcoxon rank sum test was used in order to identify if for each set of texture features extracted from the middle component of the CCA plaques, a significant difference (S) or not (NS) exists, with a confidence level of 95\%. For significant differences, we require $\mathrm{p}<0.05$. Furthermore, box plots for the different texture features, were plotted. Bland-Altman plots [20], with $95 \%$ confidence intervals, were also used to further evaluate the differences between texture features extracted from the asymptomatic and symptomatic CCA plaques. Also, the correlation coefficient, $\rho$, between the aforementioned textures features were investigated, which reflects the extent of a linear relationship between two data sets.

\section{$3 \quad$ Results}

Figure 1 illustrates a manually segmented asymptomatic (see left column of Fig. 1) and a symptomatic (see right column of Fig. 1) plaque from an ultrasound image of the CCA. We also present the normalized image (see Fig. 1b), normalized despeckled images, (see Fig. 1c) and the three different plaque components (see Fig. 1d), respectively.

Table 1 presents selected texture features (mean \pm IQR) extracted from the middle component of the plaques, that showed significant differences between the asymptomatic and symptomatic CCA plaques for the original and despeckled images (- / -). The IQR values for each feature are given in parentheses $( \pm I Q R)$. The nonparametric Wilcoxon rank-sum test was performed between the asymptomatic and symptomatic despeckled CCA plaques and the p-values are given in the last column of Table 1, showing statistical significant differences between the two different groups.

In Fig. 2 we present box plots for selected plaque texture features that showed significant differences between asymptomatic and symptomatic subjects, extracted from the middle component of the CCA plaques from all subjects for the original and the despeckled images with filter DsFlsmv. We show the texture features: a) mean and sum average (SAV) for asymptomatic (_A) and symptomatic (_S) subjects as well as for the original and the despeclked (_d) images.

The correlation coefficient, $\rho$, between the texture feature Mean_A and Mean_S for the original images was $\rho=0.034(\mathrm{p}=0.74)$, and between the Mean_Ad and Mean_Sd for the despeckled images was $\rho=0.0037(\mathrm{p}=0.94)$. The correlation coefficient, $\rho$, between the texture feature SAV_A and SAV_S for the original images was $\rho=0.04$ $(\mathrm{p}=0.65)$, and between the SAV_Ad and SAV_Sd for the despeckled images was $\rho=0.007(p=0.94)$. 


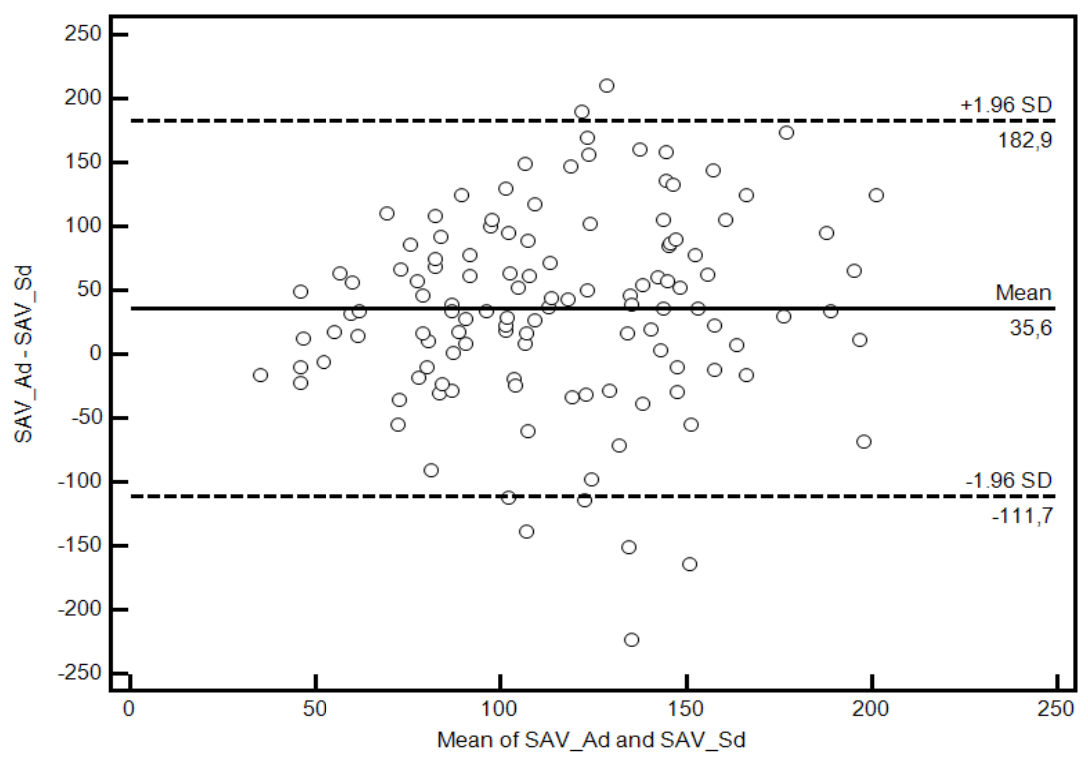

Fig. 3. Regression lines (Bland-Altman plot) between the texture feature sum average for the asymptmatic and the symptomatic normalised despeckled images. The middle line represents the mean difference, and the upper and lower two outside lines representthe limits of agreement between the two measurents, which are the mean of the data \pm 2 sd for the estimated difference between the two measurements.

Figure 3 presents a Bland-Altman plot for the texture feature sum average (SAV) extracted from the middle component of the carotid plaque for the asymptomatic and the symptomatic normalised despeckled images for all subjects investigated in this study. The difference of the two measurements between the asymptomatic and symptomatic images was 35.6+182.9 and 35.6-111.7 $(\rho=0.007$ at $p=0.94)$.

\section{Discussion and Conclusions}

In this study, we use the manual segmentations from a neurovascular expert to perform texture analysis of the middle component of the atherosclerotic carotid plaque in ultrasound images of the CCA, in 115 asymptomatic and 115 symptomatic subjects. Our objective in this study was to estimate significant differences between the aforementioned groups of patients which may lead to a more accurate evaluation of the risk of stroke. The study showed that there are some texture features that might be used to classify the two different groups of subjects as well as to follow up the progression of the atherosclerosis disease. The findings of the present study may be helpful in understanding the development of stroke in asymptomatic and/or symptomatic subjects at risk of atherosclerosis. We showed in the present study, that the middle component of the atherosclerotic carotid plaque in ultrasound images may be helpful in classifying, possibly more reliably, asymptomatic and symptomatic subjects and might contribute to the research for the evaluation of the risk of stroke and its association with the vulnerable atherosclerotic carotid plaque. We also found strong statistical significant differences for 
the asymptomatic and the symptomatic subjects for the most of the 71 different texture features investigated in the present study.

There are no other studies reported in the literature where the middle component, or other parts, of the atherosclerotic carotid plaque in ultrasound images of the CCA was investigated for evaluating the risk of stroke in asymptomatic and/or symptomatic subjects at risk of atherosclerosis. There is only one cross-sectional clinical study [7], consisting of patients with asymptomatic and symptomatic plaques found in the literature, where the association between the presence of juxtaluminal hypoechoic plaque areas without a visible echogenic cap and symptoms was found. The results in [7], confirmed earlier clinical observations and indicate for the first time an $8 \mathrm{~mm}^{2}$ cut-off point for the visible echohenic cap. This cut-off point needs to be validated by other studies and different ultrasonic equipment and then applied to prospective studies of asymptomatic patients. In [9] texture features were extracted from asymptomatic and symptomatic subjects at risk of atherosclerosis, where features were found which may classify these two different groups of subjects. In [10], morphological features extracted from atherosclerotic carotid plaques were found, that can be used for classifying plaques in asymptomatic and symptomatic.

For the initiation of this study we mainly followed unpublished results and clinical observations proposing, that different components of the atherosclerotic carotid plaque, may contribute differently to the development of the risk of stroke. We have concentrated our current investigation in the middle component of the plaque, which according to unpublished results may contribute stronger to the development of a neurological episode in asymptomatic or symptomatic subjects at risk of stroke. To overcome much of the subjectivity for plaque characterization, image normalization has been proposed using linear scaling and two reference points: blood and adventitia [12]. Various studies have validated this method for image normalization with measurement of reproducibility of the overall plaque echodensity using the gray-scale median [4]-[12] and two subsequent studies have demonstrated that the risk of stroke increases with decreased plaque gray scale median.

A limitation of our study is the lack of precise information on the duration between the onset of symptoms and the time of the ultrasound examination in individual symptomatic patients. It is therefore possible that plaque image appearance may have changed during this interval as a result of medical intervention. Additionally, the study should be further applied on a larger sample of subjects, a task which is currently undertaken by our group.

In a future study, additional variables, such as age, sex, weight, blood pressure and others should be taken into account for better evaluating the risk of stroke in the aforementioned group of patients. Furthermore, a classifier may be employed in order to provide a classification score that could provide a better insight of the best features to select for asymptomatic and symptomatic subjects.

\section{References}

1. Mendis, S., Puska, P., Norrving, B.: Global Atlas on cardiovascular disease prevention and control. WHO (2012) ISBN 978-92-4-156437-3

2. Executive committee for the asymptomatic carotid atherosclerosis study: Endarterectomy for asymptomatic carotid stenosis. J. Am. Med. Assoc. 273, 142-1428 (2002) 
3. Nicolaides, A.N., Sabetai, M., Kakkos, S.K., Dhanjil, S., Tegos, T., Stevens, J.M.: The asymptomatic carotid stenosis and risk of stroke study. Int. Angiol. 22(3), 263-272 (2003)

4. Loizou, C.P., Pattichis, C.S., Pantziaris, M., Nicolaides, A.N.: An integrated system for the segmentation of atherosclerotic carotid plaque. IEEE Trans. Inform. 11(6), 661-667 (2007)

5. Loizou, C.P., Kasparis, T., Spyrou, C., Pantziaris, M.: Integrated system for the complete segmentation of the carotid artery bifurcation in ultrasound images. In: 9th Int Conf. Artif. Intell. Applic. \& Innov. (AIAI 2013), Pafos, Cyprus, September 26-28, pp. 1-10 (2013)

6. Loizou, C.P., Pattichis, C.S., Petroudi, S., Kasparis, T., Pantziaris, M., Nicolaides, A.N.: Segmentation of atherosclerotic carotid plaque in ultrasound video. In: 34th Ann. Int. Conf. IEEE Eng. Med. Biol., San Diego, USA, August 28-September1, pp. 53-56 (2012)

7. Griffin, M., Kyriakou, E., Pattichis, C.S., Bond, D., et al.: Juxtaluminal hypoechoic area in ultrasonic images of carotid plaques and hymispheric symptoms. J. Vasc. Surg. 52(1), 69-76 (2010)

8. Geroulakos, G., Ramaswami, G., Nicolaides, A.N., James, K., Labropoulos, N., Belcaro, G., et al.: Characterization of symptomatic and asymptomatic carotid plaques using high-resolution real-time ultrasonography. Br. J. Surg. 80, 1274-1277 (1993)

9. Christodoulou, C., Pattichis, C.S., Pantziaris, M., Nicolaides, A.N.: Texture-based classification of atherosclerotic carotid plaques. IEEE Trans. Med. Imag. 22, 902-912 (2003)

10. Kyriakou, E., Pattichis, M.S., Christodoulou, C.I., Pattichis, C.S., et al.: Ultrasound imaging in the analysis of carotid plaque morphology for the assessment of stroke. In: Suri, J.S., Yuan, C., Wilson, D.L., Laxminarayan, S. (eds.) Plaque Imaging: Pixel to Molecular level, pp. 241-275. IOS Press (2005)

11. A Philips Medical System Company. Comparison of image clarity, SonoCT real-time compound imaging versus conventional 2D ultrasound imaging. ATL Ultrasound, Report (2001)

12. Elatrozy, T., Nicolaides, A.N., Tegos, T., Zarka, A., Griffin, M., Sabetai, M.: The effect of B-mode ultrasonic image standardization of the echodensity of symptomatic and asymptomatic carotid bifurcation plaque. Int. Angiol. 17(3), 179-186 (1998)

13. Lee, J.S.: Digital image enhancement and noise filtering by using local statistics. IEEE Trans. Pattern Anal. Mach. Intellig. PAMI-2(2), 165-168 (1980)

14. Loizou, C.P., Pattichis, C.S., Christodoulou, C.I., Istepanian, R.S.H., Pantziaris, M., Nicolaides, A.: Comparative evaluation of despeckle filtering in ultrasound imaging of the carotid artery. IEEE Trans. Ultras. Ferroel. Freq. Contr. 52(10), 1653-1669 (2005)

15. Haralick, R.M., Shanmugam, K., Dinstein, I.: Texture features for image classification. IEEE Trans. Systems, Man., Cyber. SMC-3, 610-621 (1973)

16. Weszka, J.S., Dyer, C.R., Rosenfield, A.: A comparative study of texture measures for terrain classification. IEEE Trans. Syst. Man. Cyber. SMC-6, 269-285 (1976)

17. Amadasun, M., King, R.: Textural features corresponding to textural properties. IEEE Trans. Syst. Man. Cyber. 19(5), 1264-1274 (1989)

18. Wu, C.M., Chen, Y.C., Hsieh, K.-S.: Texture features for classification of ultrasonic images. IEEE Trans. Med. Imag. 11, 141-152 (1992)

19. Chen, T.-J., et al.: A novel image quality index using Moran I statistics. Ph. in Medic. Biol. 48, 131-137 (2003)

20. Bland, J.M., Altman, D.G.: Statistical methods for assessing agreement between two methods of clinical measurement. Lancet 1(8476), 307-310 (1986) 